\title{
On the thermal-hydraulic optimization of DEMO divertor plasma facing components cooling circuit
}

\author{
P. A. Di Maio ${ }^{a^{*}}$, S. Garitta ${ }^{\mathrm{a}}$, J. H. You ${ }^{\mathrm{b}}$, G. Mazzone ${ }^{\mathrm{c}}$, M. Marino ${ }^{\mathrm{a}}$, E. Vallone ${ }^{\mathrm{a}}$ \\ ${ }^{a}$ University of Palermo, Viale delle Scienze, Edificio 6, 90128 Palermo, Italy \\ ${ }^{b}$ Max Planck Institute of Plasma Physics (E2M), Boltzmann Str.2, 85748 Garching, Germany \\ ${ }^{c}$ Department of Fusion and Technology for Nuclear Safety and Security, ENEA C.R. Frascati, via E. Fermi 45, 00044 \\ Frascati (Roma), Italy
}

Within the framework of the Work Package Divertor, Subproject: Cassette Design and Integration (WPDIVCassette) of the EUROfusion action, a research campaign has been jointly carried out by ENEA and University of Palermo to investigate the thermal-hydraulic performances of the DEMO divertor cassette cooling system.

Attention has been focussed on the divertor Plasma Facing Components (PFCs) cooling circuit and a parametric analysis has been carried out in order to assess the potential impact of proper layout changes on its thermal-hydraulic performances, mainly in terms of coolant total pressure drop, flow velocity distribution and margin against critical heat flux occurrence.

The research activity has been carried out following a theoretical-computational approach based on the finite volume method and adopting a qualified Computational Fluid-Dynamic (CFD) code. Results obtained have allowed to select a revised PFCs cooling circuit configuration, suitable to comply with the prescribed thermalhydraulic limits assumed for the DEMO divertor design. They are reported and critically discussed.

Keywords: DEMO, divertor, plasma facing components, CFD analysis, thermofluid-dynamics.

\section{Introduction}

The recent European Fusion Development Agreement roadmap was drafted to realize commercially viable fusion power generation [1]. Within this framework, the divertor is a key in-vessel component, being responsible for power exhaust and impurity removal via guided plasma. Due to its position and functions, the divertor has to sustain very high heat and particle fluxes arising from the plasma (up to 20 $\mathrm{MW} / \mathrm{m}^{2}$ ), while experiencing an intense nuclear deposited heat power, which could jeopardize its structure and limit its lifetime. Therefore, attention has to be paid to the thermal-hydraulic design of its cooling system to ensure a uniform and proper cooling, providing a safe margin against Critical Heat Flux (CHF) without an unduly high pressure drop [2,3].

Within the framework of the activities foreseen by the Work Package Divertor, Subproject: Cassette Design and Integration (WPDIV-Cassette) of the EUROfusion action, a research campaign has been carried out at the University of Palermo, in cooperation with ENEA, to investigate the potential improvements of Plasma Facing Components (PFCs) cooling circuit thermal-hydraulic performances due to proper changes of its configuration $[3,4]$. Their aptitude to enhance PFCs cooling circuit thermal-hydraulic performances, mainly in terms of reduction of coolant total pressure drop and flattening of flow velocity and CHF margin distributions along Plasma Facing Unit (PFU) channels, has been assessed under nominal steady state conditions, checking whether the corresponding reference limits, namely the maximum coolant total pressure drop (1.4 MPa) and the minimum margin against CHF onset (1.4) at strike point sections of Vertical Targets (VTs) PFU channels result to be met.
The research campaign has been carried out following a theoretical-computational approach based on the Finite Volume Method and adopting the commercial Computational Fluid-Dynamic (CFD) code ANSYS CFX v.16.2, employed also to evaluate concentrated hydraulic resistances to be used in system codes $[5,6,7]$. Analysis models and assumptions are herein reported and critically discussed, together with the main results obtained.

\section{Outline of DEMO divertor cassette}

According to its 2016 design, DEMO divertor is articulated in 54 toroidal cassettes, each composed of a Cassette Body (CB) supporting two PFCs, namely an Inner Vertical Target (IVT) and an Outer Vertical Target (OVT) (Fig. 1), composed of actively cooled PFUs equipped with a Swirl Tape (ST) turbulence promoter.

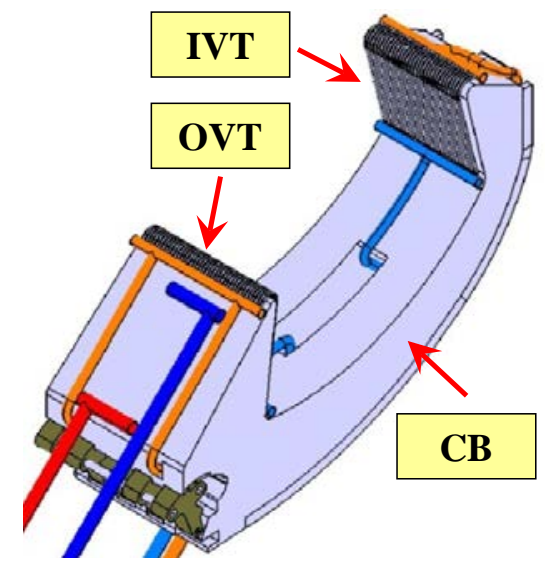

Fig. 1. DEMO divertor cassette 2016 design. 


\section{PFCs cooling circuit}

The PFCs cooling circuit layout option 2 (Fig. 2) has been selected as the most promising among those considered during 2015 research campaign [2,3]. During the second half of 2016 it has undergone a revision [8], mainly related to the number of PFUs within each VT (increased from 29 to 31 in the IVT and from 37 to 39 in the OVT) and to the OVT manifolds lay-out (including two separate outlet manifolds instead of a single one). The revised lay-out (Rev A) has, hence, been adopted as reference configuration for this optimization study.

It relies on the use of subcooled pressurized water at the inlet pressure and temperature of $5 \mathrm{MPa}$ and $130^{\circ} \mathrm{C}$, respectively, flowing under quasi-isothermal conditions.

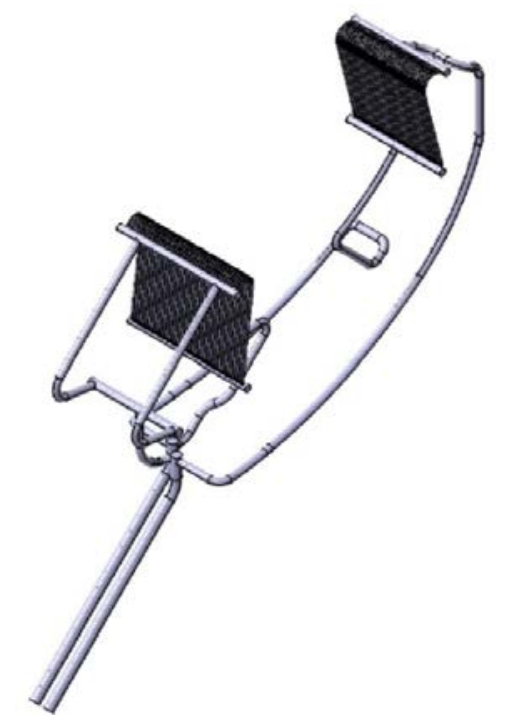

Fig. 2. PFCs cooling circuit layout option 2 (Rev A).

\section{PFCs cooling circuit CFD analysis}

In the first phase of the research campaign, the thermal-hydraulic performances of the considered PFCs cooling circuit (Rev A) have been assessed by running a steady state, isothermal CFD analysis under the coolant operative conditions agreed in August 2016 with EUROfusion teams (Table 1).

Table 1. Summary of coolant operative conditions.

\begin{tabular}{lcc}
\hline & $\begin{array}{c}\text { August } \\
\mathbf{2 0 1 6}\end{array}$ & $\begin{array}{c}\text { October } \\
\mathbf{2 0 1 6}\end{array}$ \\
\hline Inlet Pressure [MPa] & 5.0 & 5.0 \\
Inlet Temperature $\left[{ }^{\circ} \mathbf{C}\right]$ & 130 & 130 \\
$\Delta \mathbf{T}\left[{ }^{\circ} \mathbf{C}\right]$ & 6 & 6 \\
Removed Power $[\mathbf{M W}]$ & 126 & 136 \\
G per Cassette $[\mathbf{k g} / \mathbf{s}]$ & 91.37 & 98.62 \\
\hline
\end{tabular}

Selected mesh parameters and main assumptions, models and Boundary Conditions (BCs) adopted are reported in Tables 2 and 3, respectively.
Table 2. Summary of selected mesh parameters.

\begin{tabular}{lc}
\hline Nodes & $9.667 \cdot 10^{+6}$ \\
Elements & $2.144 \cdot 10^{+7}$ \\
Inflation layers number & 12 \\
First layer thickness $[\boldsymbol{\mu m}]$ & 10 \\
Layers growth rate & 1.5 \\
Typical element size [m] & $2.75 \cdot 10^{-3}$ \\
Surface with $\mathbf{y}^{+}<\mathbf{1 3 0}[\%]$ & 95.8 \\
Model simplification & No ST \\
\hline
\end{tabular}

Table 3. Summary of assumptions, models and BCs.

\begin{tabular}{lc}
\hline Analysis type & Steady state \\
Material library & IAPWS IF97 \\
Temperature & $133^{\circ} \mathrm{C}$ \\
Turbulence model & $\mathrm{k}-\varepsilon$ \\
Boundary layer modelling & Scalable wall functions \\
Wall roughness & $15 \mu \mathrm{m}$ \\
Inlet BC & $\mathrm{p}_{\mathrm{s}}=5 \mathrm{MPa}$ \\
Outlet BC & $\mathrm{G}=91.37 \mathrm{~kg} / \mathrm{s}$ \\
\hline
\end{tabular}

The results of the PFCs cooling circuit CFD analysis under nominal steady state conditions, widely reported in [4], have allowed to conclude that:

- total pressure drop amounts to $2.98 \mathrm{MPa}$, being more than the double of prescribed limit (1.4 MPa);

- flow velocity distribution within PFU channels is extremely uneven with deviations between maximum and minimum values higher than $40 \%$;

- CHF margin is predicted to stay below 1.4 within several channels of IVT and OVT PFUs.

Therefore, a deep review of the cooling circuit configuration has been launched intended to improve its thermal-hydraulic performances, reducing total pressure drop and flattening flow velocity and CHF margin distributions so to fulfil their pertaining requirements.

\section{PFCs cooling circuit optimization}

In the second phase of the research activity, an optimisation study has been performed to investigate the potential improvements of PFCs cooling circuit thermalhydraulic performances due to proper changes of its configuration [4]. To this purpose, a parametric study has been carried out to numerically assess the effects of:

- increasing VTs inlet/outlet manifolds diameters;

- placing a properly-shaped diffuser at the inlet section of each VT inlet toroidal header.

In particular, the increase of manifolds diameters aims to reduce those distributed hydraulic resistances that heavily contribute to total pressure drop, while the introduction of a proper diffuser between each VT inlet manifold and header aims to flatten both flow velocity and CHF margin distributions along PFU channels. 
To this purpose, some revised configurations of the PFCs cooling circuit reference lay-out (Rev A) have been considered, characterized by manifolds diameters increased by a factor 1.2 (Rev B in Fig. 3) and 1.4 (Rev $\mathrm{C}$ in Fig. 4) and by VTs diffusers with lengths and widths (Fig. 5) reported in Table 4.

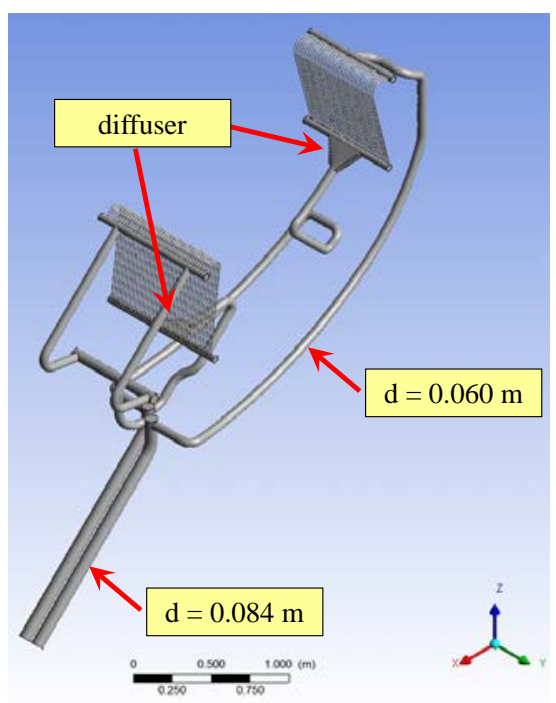

Fig. 3. PFCs cooling circuit configuration Rev B1.

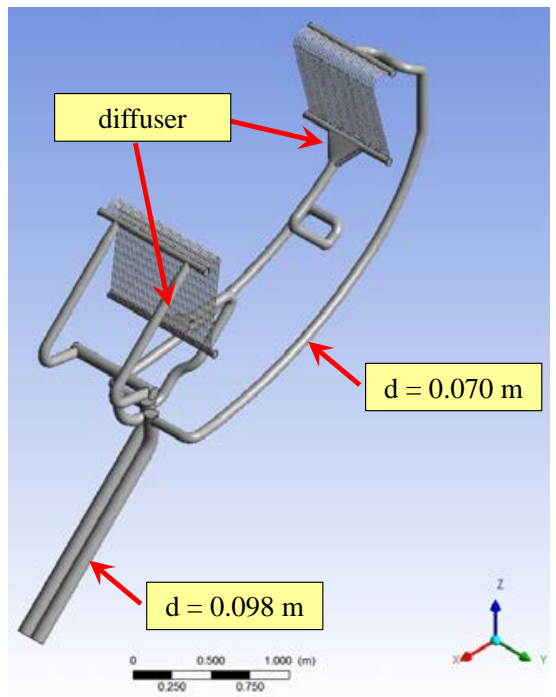

Fig. 4. PFCs cooling circuit configuration Rev C1.

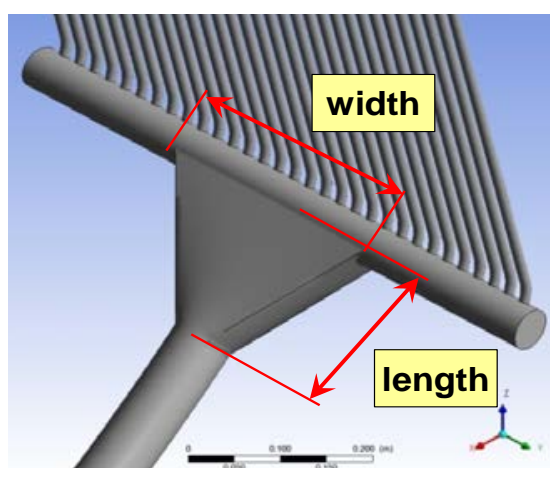

Fig. 5. VT diffuser geometrical parameters.
Table 4. PFCs cooling circuit configurations investigated.

\begin{tabular}{lcc}
\hline \multirow{2}{*}{ Rev } & \multicolumn{2}{c}{ Diffuser } \\
\cline { 2 - 3 } & Length [cm] & Width [cm] \\
\hline B1 & 18 (OVT) - 19 (IVT) & 30 \\
B2 & 18 (OVT) - 19 (IVT) & 60 \\
B3 & 39 (OVT) - 33 (IVT) & 30 \\
B4 & 39 (OVT) - 33 (IVT) & 60 \\
C1 & $18($ OVT) - 19 (IVT) & 30 \\
C2 & $18($ OVT) - 19 (IVT) & 60 \\
C3 & 39 (OVT) - 33 (IVT) & 30 \\
C4 & 39 (OVT) - 33 (IVT) & 60 \\
\hline
\end{tabular}

The steady state thermal-hydraulic performances of the considered cooling circuit configurations have been investigated by running eight dedicated isothermal CFD analyses with the ANSYS CFX v.16.2 code. To this purpose, mesh parameters analogous to those of Table 2 have been assumed, while main assumptions, models and BCs reported in Table 3 have been adopted.

Results obtained in terms of total pressure, flow velocity and CHF margin distributions are reported and critically discussed in the following subsections.

\subsection{Results: total pressure drop}

The spatial distribution of total pressure field, calculated for the reference configuration is reported in Fig. 6, the others obtained being quite similar.

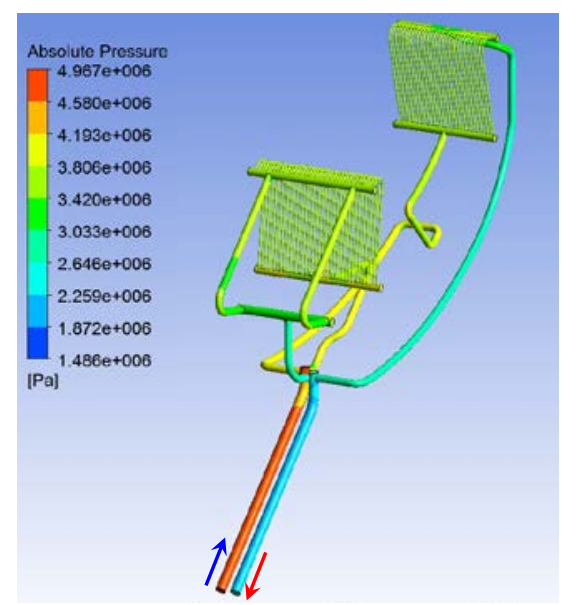

Fig. 6. Total pressure field distribution (Rev A).

Since no swirl tapes have been simulated to speed-up calculations, the assessed total pressure drops $\left(\Delta \mathrm{p}_{\text {No ST }}\right)$ have been corrected to avoid their underestimation [2]. To this purpose, the increase in pressure drop due to STs $\left(\Delta \mathrm{p}_{\mathrm{ST}}\right)$ has been estimated, according to the correlation given in [9], with reference to the PFU cooling channel where the highest mass flow rate is predicted. A more detailed description may be found in [4].

The overall total pressure drops estimated for the investigated configurations $(\Delta \mathrm{p})$ are reported in Table 5 . 
Table 5. PFCs cooling circuits total pressure drops [MPa].

\begin{tabular}{lccc}
\hline & $\Delta \mathbf{p}_{\text {No ST }}$ & $\Delta \mathbf{p}_{\text {ST }}$ & $\Delta \mathbf{p}$ \\
\hline Rev A & 2.612 & 0.368 & 2.979 \\
Rev B1 & 1.195 & 0.244 & 1.439 \\
Rev B2 & 1.213 & 0.259 & 1.472 \\
Rev B3 & 1.167 & 0.216 & 1.383 \\
Rev B4 & 1.216 & 0.237 & 1.453 \\
Rev C1 & 0.730 & 0.209 & 0.939 \\
Rev C2 & 0.739 & 0.211 & 0.950 \\
Rev C3 & 0.736 & 0.189 & 0.926 \\
Rev C4 & 0.742 & 0.205 & 0.947 \\
\hline
\end{tabular}

Results show that the total pressure drops calculated for Rev B configurations are slightly higher than the prescribed limit (1.4 MPa), except for Rev B3, where due to a "long" diffuser with a mild transition in width, flow expansion takes place inside the diffuser without significant turbulent recirculation regions (Fig. 7), reducing the total pressure drop within the limit, even if with a small margin of $0.017 \mathrm{MPa}$. Conversely, the total pressure drops calculated for Rev $\mathrm{C}$ configurations result significantly lower than $1.4 \mathrm{MPa}$, with a margin of at least $0.45 \mathrm{MPa}$ (Rev C2), mainly due to the manifold diameter increase up to 1.4 times its original value [4].

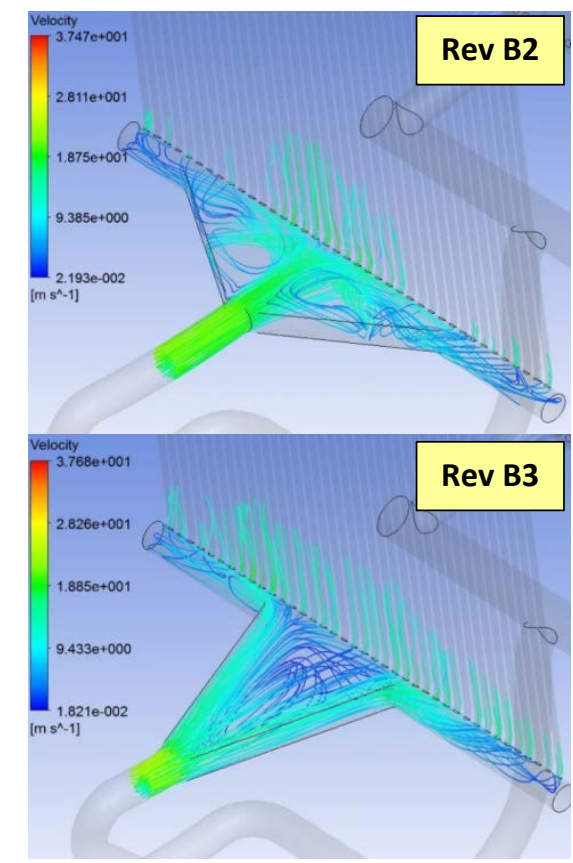

Fig. 7. Streamlines in the diffuser (Rev B2 vs B3).

Moreover, results indicate that the diffuser shape has a slight impact on the total pressure drop, mainly due to its aptitude to reduce turbulent recirculation regions, where pressure dissipation occurs, at the connection between VTs manifolds and their inlet headers. In fact, pressure drops range from 1.472 $\mathrm{MPa}(\mathrm{Rev} \mathrm{B} 2)$ to 1.383 $\mathrm{MPa}$ (Rev B3) and from 0.950 MPa (Rev C2) to 0.926 $\mathrm{MPa}$ (Rev C3) in case of Rev B and Rev C configurations, respectively. In particular, as to Rev B configurations, the increase in diffuser width induces a slight raise of total pressure drop of $\approx 5 \%$ at most (Rev B3 vs Rev B4). Furthermore, the increase in diffuser length determines a slight reduction of total pressure drop of $\approx 4 \%$ at most (Rev B1 vs Rev B3). Similarly, as to Rev C configurations, the increase in diffuser width induces a slight raise of total pressure drop of $\approx 3 \%$ at most (Rev C3 vs Rev C4) and the increase in diffuser length determines a slight reduction of total pressure drop of $\approx 2 \%$ at most ( Rev C1 vs Rev C3).

\subsection{Results: flow velocity}

The axial flow velocity distributions along VTs PFU channels have been assessed in presence of STs, to check whether unbalanced distributions might take place, preventing a uniform cooling of solid components. Results indicate the occurrence of non-uniform axial flow velocity distributions for all the configurations studied, mainly due to the by-pass effect across horizontal headers induced by VTs manifolds whose impact seems to be mitigated by the diffusers. Attention has, hence, been paid to axial flow velocity distributions along OVT PFU channels (Figs. 8-9 and Table 6), since they experience the worst conditions.

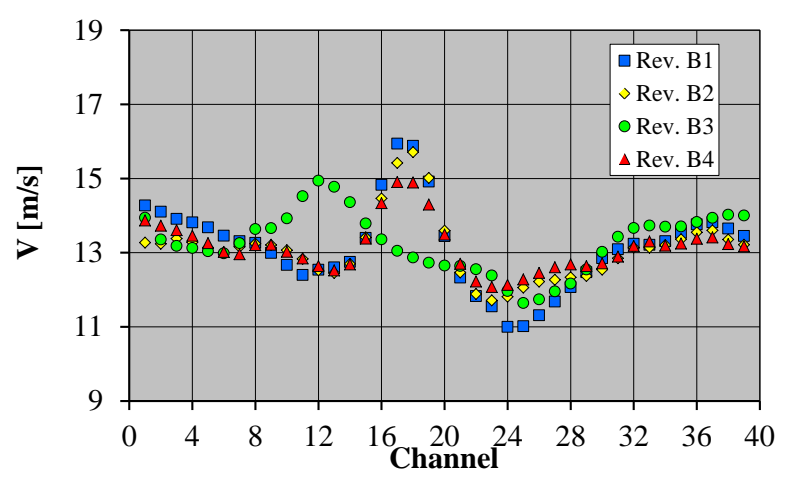

Fig. 8. OVT axial flow velocity distributions - Rev B.

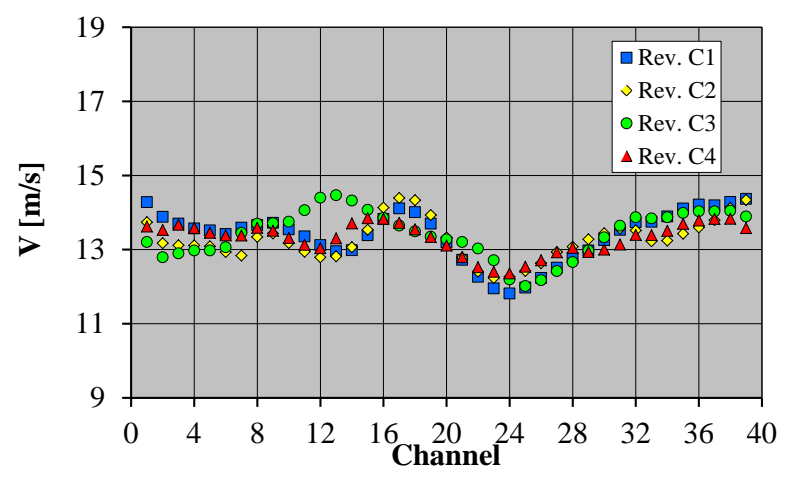

Fig. 9. OVT axial flow velocity distributions - Rev C.

Results indicate that the diffuser highly contributes to flattening the axial flow velocity distributions along VTs PFU channels, with performances strongly depending on its configuration. In fact, the deviation, $\varepsilon_{\mathrm{V}}$, between maximum and minimum channel velocities undergoes 
strong variations due to change in diffuser length and width. In particular, as to Rev $\mathrm{B}$ configurations, the increase in diffuser width induces a decrease of $\varepsilon_{\mathrm{V}}$ of $\approx 18 \%$ at most (Rev B1 vs Rev B2). Furthermore, the increase in diffuser length determines a more pronounced reduction of $\varepsilon_{\mathrm{V}}$ amounting to $\approx 29 \%$ (Rev B1 vs Rev B3). Similarly, as to Rev C configurations, the increase in diffuser width induces a sharp decrease of $\varepsilon_{\mathrm{V}}$ of $\approx 36 \%$ at most ( Rev C3 vs Rev C4) and the increase in diffuser length determines a reduction of $\varepsilon_{\mathrm{V}}$ of $\approx 28 \%$ at most (Rev C2 vs Rev C4).

Table 6. OVT axial flow velocity distribution data.

\begin{tabular}{|c|c|c|c|}
\hline & $\mathrm{V}_{\text {Max }}[\mathrm{m} / \mathrm{s}]$ & $\mathrm{V}_{\min }[\mathrm{m} / \mathrm{s}]$ & $\varepsilon_{V}[\%]$ \\
\hline $\operatorname{Rev} A$ & 20.867 & 9.400 & 54.95 \\
\hline Rev B1 & 15.940 & 10.998 & 31.01 \\
\hline Rev B2 & 15.712 & 11.711 & 25.46 \\
\hline Rev B3 & 14.941 & 11.640 & 22.09 \\
\hline Rev B4 & 14.907 & 12.074 & 19.00 \\
\hline Rev C1 & 14.368 & 11.816 & 17.76 \\
\hline Rev C2 & 14.391 & 12.232 & 15.00 \\
\hline Rev C3 & 14.466 & 12.016 & 16.94 \\
\hline Rev C4 & 13.847 & 12.355 & 10.78 \\
\hline
\end{tabular}

\subsection{Results: CHF margin}

The distributions of the margin, $\mathrm{M}$, against $\mathrm{CHF}$ onset within the VTs PFU channels have been assessed for the considered PFCs cooling circuit configurations, to check whether the prescribed minimum value of 1.4 is guaranteed. To this purpose, the procedure described in [2] has been followed and the axial flow velocity predicted for each swirled channel has been used in input to the correlation given in [9] to calculate the pertaining CHF value. This has been compared to the normal heat flux estimated at the wall-coolant interface due to the peak heat flux expected at the strike points, to finally assess the margin, $\mathrm{M}$, against thermal crisis occurrence.

Attention has been paid to the distributions along OVT PFU channels (Table 7 and Figs. 10-11), since they experience the highest peak heat flux of $20 \mathrm{MW} / \mathrm{m}^{2}$.

Table 7. OVT CHF margin distribution data.

\begin{tabular}{lccc}
\hline & $\mathbf{M}_{\text {Max }}$ & $\mathbf{M}_{\min }$ & $\boldsymbol{\varepsilon}_{\mathbf{M}}[\%]$ \\
\hline $\operatorname{Rev}$ A & 1.826 & 1.070 & 41.42 \\
$\operatorname{Rev}$ B1 & 1.656 & 1.283 & 22.53 \\
$\operatorname{Rev~B2}$ & 1.637 & 1.336 & 18.40 \\
$\operatorname{Rev}$ B3 & 1.583 & 1.337 & 15.55 \\
$\operatorname{Rev}$ B4 & 1.580 & 1.367 & 13.47 \\
$\operatorname{Rev}$ C1 & 1.577 & 1.382 & 12.35 \\
$\operatorname{Rev}$ C2 & 1.583 & 1.414 & 10.67 \\
$\operatorname{Rev}$ C3 & 1.588 & 1.398 & 11.96 \\
$\operatorname{Rev}$ C4 & 1.540 & 1.424 & 7.51 \\
\hline
\end{tabular}

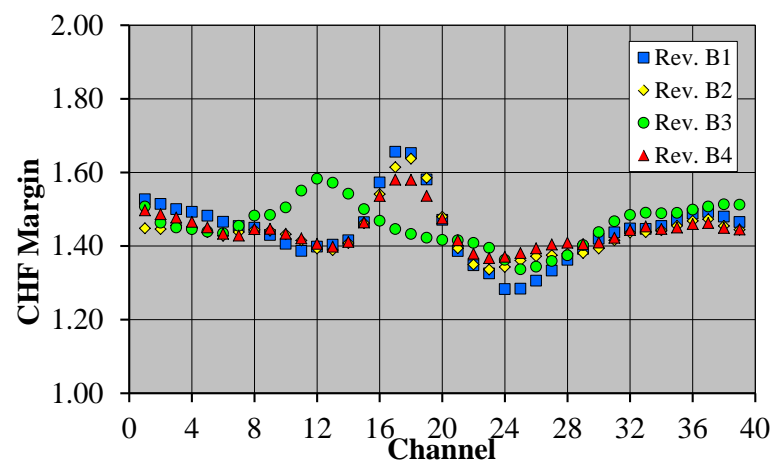

Fig. 10. OVT CHF margin distributions - Rev B.

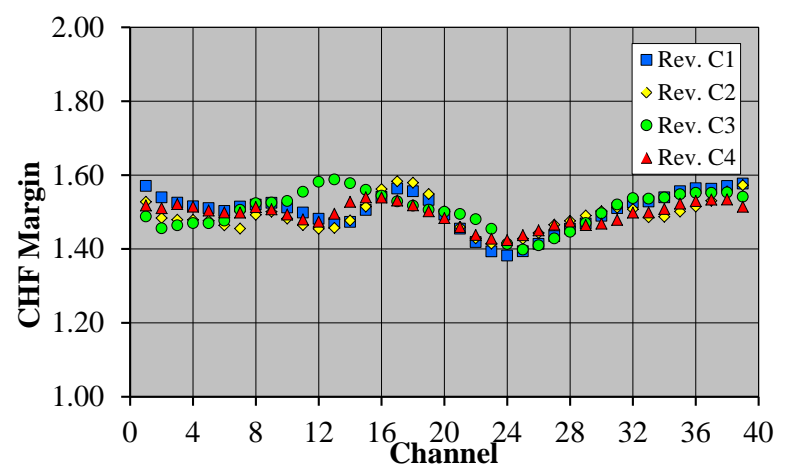

Fig. 11. OVT CHF margin distributions - Rev C.

As expected, results confirm that the diffuser significantly contribute also to flattening the CHF margin distributions along VTs PFU channels, with an effectiveness strongly depending on its configuration. In fact, the deviation, $\varepsilon_{\mathrm{M}}$, between maximum and minimum margins undergoes strong variations due to change in diffuser length and width. In particular, as to Rev B configurations, the increase in diffuser width induces an $\varepsilon_{\mathrm{M}}$ decrease of $\approx 18 \%$ at most (Rev B1 vs Rev B2), while the increase in its length determines an $\varepsilon_{\mathrm{M}}$ reduction of $\approx$ 31\% (Rev B1 vs Rev B3). Similarly, as to Rev C configurations, the increase in diffuser width induces a strong $\varepsilon_{\mathrm{M}}$ decrease of $\approx 37 \%$ at most (Rev C3 vs Rev $\mathrm{C} 4$ ), while the increase in its length determines a marked $\varepsilon_{\mathrm{M}}$ reduction of $\approx 30 \%$ at most (Rev C2 vs Rev C4). Finally, it has to be underlined that the minimum CHF margins calculated for the OVT PFU channels, in case of Rev B configurations, result slightly lower $(\leq 8 \%)$ than the prescribed limit of 1.4 , while, as to Rev C configurations, they result slightly higher $(\approx 1 \div 2 \%)$, in case of Rev C2 and Rev C4 configurations, and slightly lower, for Rev C1 and Rev C3 ( $\leq 1 \%)$ configurations.

\subsection{Conclusions of the optimization study}

The optimization study has allowed selecting the Rev C4 as the most effective PFCs cooling circuit configuration from the thermal-hydraulic standpoint, since it maximizes the performances while fulfilling all the prescribed requirements in terms of total pressure drop ( $<1.4 \mathrm{MPa}$ ) as well as of axial flow velocity $(<16$ $\mathrm{m} / \mathrm{s}$ ) and CHF margin ( $>1.4)$ along PFU channels. 


\section{Optimized PFCs cooling circuit CFD analysis}

In the third phase of the research campaign, attention has been focussed on the Rev C4 PFCs cooling circuit optimized configuration and its pertaining thermalhydraulic performances have been assessed by running a steady state, isothermal CFD analysis under the coolant operative conditions agreed in October 2016 with EUROfusion teams (Table 1). The mesh parameters selected are reported in Table 8 while the main assumptions, models and BCs adopted are those reported in Table 3, with the only exception of the outlet BC, for which a mass flow rate amounting to $98.62 \mathrm{~kg} / \mathrm{s}$ instead of $91.37 \mathrm{~kg} / \mathrm{s}$ has been assumed.

Table 8. Summary of selected mesh parameters.

\begin{tabular}{lc}
\hline Nodes & $1.109 \cdot 10^{+7}$ \\
Elements & $2.499 \cdot 10^{+7}$ \\
Inflation layers number & 12 \\
First layer thickness $[\boldsymbol{\mu m}]$ & 10 \\
Layers growth rate & 1.5 \\
Typical element size [m] & $3.23 \cdot 10^{-3}$ \\
Surface with $\mathbf{y}^{+}<\mathbf{1 3 0}[\%]$ & 96.5 \\
Model simplification & No ST \\
\hline
\end{tabular}

The results of the optimized PFCs cooling circuit CFD analysis under nominal steady state conditions, widely reported in [4], are summarized in Table 9.

Table 9. Summary of CFD analysis results.

\begin{tabular}{lc}
\hline $\mathbf{G}[\mathrm{kg} / \mathrm{s}]$ & 98.62 \\
$\Delta \mathbf{p}[\mathrm{MPa}]$ & 1.098 \\
Pumping power [kW] & 115.9 \\
$\mathbf{V}_{\text {Max }}$ - OVT channels [m/s] & 14.935 \\
$\mathbf{V}_{\min }$ - OVT channels [m/s] & 13.434 \\
$\mathbf{M}_{\text {Max }}$ - OVT channels & 1.574 \\
$\mathbf{M}_{\min }$ - OVT channels & 1.462 \\
$\mathbf{V}_{\text {Max }}$ - IVT channels [m/s] & 16.896 \\
$\mathbf{V}_{\text {min }}$ - IVT channels [m/s] & 13.637 \\
$\mathbf{M}_{\text {Max }}$ - IVT channels & 1.696 \\
$\mathbf{M}_{\min }$ - IVT channels & 1.469 \\
\hline
\end{tabular}

Results indicate that total pressure drop increases of $\approx 16 \%$ from the previously calculated value of 0.947 $\mathrm{MPa}$ to $1.098 \mathrm{MPa}$, remaining widely below the limit of 1.4 MPa. This is as an obvious consequence of the mass flow rate increase from $91.37 \mathrm{~kg} / \mathrm{s}$ up to $98.62 \mathrm{~kg} / \mathrm{s}$, due to the corresponding increase of total removed heat power foreseen by the operative conditions agreed in October 2016 (136 MW, see Table 1) with respect to those agreed in August 2016 (126 MW, see Table 1). Moreover, results suggest that the axial flow velocity distributions within IVT and OVT PFU channels are acceptably uniform, since maximum deviations in the order of $10 \% \div 20 \%$ have been estimated between their maximum and minimum values. Similarly, results highlight that the calculated distributions of CHF margin within VTs PFU channels are acceptably uniform, since maximum deviations between their pertaining maximum and minimum values range between $7.1 \%$ and $13.4 \%$, their minimum values resulting higher than the prescribed limit of 1.4. In conclusion, the CFD analysis of the optimised PFCs cooling circuit configuration under the reference steady state conditions agreed in October 2016 has allowed to conclude that it fulfils all the prescribed thermal-hydraulic requirements, showing a total pressure drop of $1.098 \mathrm{MPa}$, widely lower than the limit of 1.4 MPa, and a minimum margin against CHF occurrence of 1.462, higher than the limit of 1.4. Therefore, this configuration (Rev C4) is strongly encouraged for the further review of the PFCs cooling circuit design.

\section{Conclusions}

Within the framework of the activities foreseen in the WPDIV-Cassette of the EUROfusion action, a research campaign has been carried out at the University of Palermo, in cooperation with ENEA, to investigate the PFCs cooling circuit thermal-hydraulic performances and assess their potential improvement due to either the increase of manifold diameters and/or the introduction of a properly-shaped diffuser between VTs manifold and inlet headers. A theoretical-computational approach based on the Finite Volume Method has been followed and the CFD code ANSYS CFX has been adopted to carry out the optimization study. The Rev C4 cooling circuit configuration, characterized by manifold diameters increased by a factor 1.4 and a "long" diffuser with a toroidal width of $60 \mathrm{~cm}$, has been selected as the most effective one from the thermal-hydraulic standpoint. In particular, its steady state CFD analysis, under the reference conditions agreed in October 2016 with EUROfusion teams, has allowed to conclude that it fulfils all the thermal-hydraulic requirements, showing a total pressure drop of $1.098 \mathrm{MPa}$, widely lower than the limit of $1.4 \mathrm{MPa}$, and a minimum margin against $\mathrm{CHF}$ occurrence of 1.462, higher than the limit of 1.4 . Therefore, this configuration is strongly suggested for the further review of the PFCs cooling circuit design.

\section{Acknowledgments}

This work has been carried out within the framework of the EUROfusion Consortium and has received funding from the Euratom research and training programme 2014-2018 under grant agreement No 633053. The views and opinions expressed herein do not necessarily reflect those of the European Commission.

\section{References}

[1] F. Romanelli et al., Fusion Electricity - A Roadmap to the Realisation of Fusion Energy, European Fusion 
Development Agreement (EFDA), 2012, ISBN 978-3-00040720-8T.

[2] P.A. Di Maio, S. Garitta, J. H. You, G. Mazzone, E. Vallone, Thermal-hydraulic behaviour of the DEMO divertor plasma facing components cooling circuit, Fusion Engineering and Design (2017), http://dx.doi.org/10.1016/j.fusengdes.2017.02.025.

[3] J.H. You, et al., Progress in the initial design activities for the European DEMO divertor: Subproject "Cassette", Fusion Eng. Des. (2017), http://dx.doi.org/10.1016/j.fusengdes.2017.03.01.

[4] P. A. Di Maio, S. Garitta, G. Mazzone, E. Vallone, DEMO Divertor - Thermo-hydraulic assessment report 2016, Final Report on Deliverable DIV-1-T002-D006, IDM Ref. N. EFDA_D_2MMTTU.

[5] P.A. Di Maio et al., Analysis of the steady state hydraulic behaviour of the ITER blanket cooling system, Fusion Eng. Des. 98-99 (2015) 1470-1473, http://dx.doi.org/10.1016/j.fusengdes.2015.05.070.

[6] P.A. Di Maio et al., Numerical simulation of the transient thermal-hydraulic behaviour of the ITER blanket cooling system under the draining operational procedure, Fusion Eng. Des. 98-99 (2015) 1664-1667, http://dx.doi.org/10.1016/j.fusengdes.2015.01.024.

[7] P.A. Di Maio et al., On the hydraulic behaviour of ITER Shield Blocks \#14 and \#08. Computational analysis and comparison with experimental tests, Fusion Eng. Des. 109-111 (2016) 30-36.

[8] Divertor_CAD_Model_2016, EFDA_D_2MN5Y3 v1.1.

[9] A.R. Raffray et al., Critical heat flux analysis and R\&D for the design of the ITER divertor, Fusion Eng. Des. 45 (1999) 377-407. 